\title{
Análise gravimétrica dos resíduos sólidos grosseiros de unidades operacionais do sistema de esgotamento sanitário Insular, Florianópolis - SC
}

\section{Analysis of coarse solid waste from operating units of the Insular sewage system, Florianópolis - SC}

\author{
Nadine Lory Bortolotto ${ }^{1}$; Cristiano Poleto ${ }^{2}$
}

\begin{abstract}
${ }^{1}$ Aluna do Programa de Mestrado Profissional em Rede Nacional em Gestão e Regulação de Recursos Hídricos - ProfÁgua pela Universidade federal do Rio Grande do Sul - UFRGS, Porto Alegre, Rio Grande do Sul, Brasil. Orcid: 0000-0002-2490-9687 E-mail: nadinebortolotto@gmail.com

2Professor da Universidade Federal do Rio Grande do Sul - UFRGS e Instituto de Pesquisas Hidráulicas IPH, Porto Alegre, Rio Grande do Sul, Brasil. Orcid: 0000-0001-7376-1634 E-mail: cristiano.poleto@ufrgs.br
\end{abstract}

RESUMO: Este trabalho teve como objetivo identificar por meio da análise de composição gravimétrica dos resíduos sólidos retidos nos gradeamentos de algumas unidades operacionais do sistema de esgoto Insular do município de Florianópolis, Estado de Santa Catarina, Brasil. Foram escolhidas duas estações elevatórias de esgoto (Beira-Mar Norte e Hospital Universitário/Trindade). Foram adotadas as seguintes classes de resíduos sólidos: plásticos, resíduos da construção civil, animais mortos, matéria orgânica, trapos e panos, rejeitos e outros. Foram identificados os resíduos sólidos que em maiores quantidades no sistema de esgotamento sanitário podem causar danos às unidades operacionais.

Palavras-chave: Resíduos Sólidos, Sistema de Esgotamento Sanitário, Elevatórias.

ABSTRACT: This work aims to identify means of analysing the gravimetric composition of solid waste retained in the fences of some operational units of the Insular sewage system in Florianópolis, State of Santa Catarina, Brazil. Two sewage pumping stations (Beira-Mar Norte and Hospital Universitário/Trindade) were chosen. The following classes of solid waste were adopted: plastics, construction waste, dead animals, organic matter, rags and cloths, tailings and others. The solid waste observed in greater quantities in the sanitary sewage system, which may cause damage to the operational units.

Keywords: Solid Waste, Sewage System, Pumping. 
Revista Brasileira de Ciência, Tecnologia e Inovação

\section{INTRODUÇÃO}

A obtenção de qualidade ambiental e sanitária no meio urbano depende do funcionamento de uma série de infraestruturas e serviços, entre eles os serviços públicos de saneamento básico. Os sistemas de esgotamento sanitário e os serviços de manejo de resíduos sólidos urbanos são aqueles que gerenciam os efluentes e resíduos gerados diretamente pela população e pelos setores econômicos e seu funcionamento adequado é essencial para garantir a preservação ambiental, bem como mitigar ou prevenir, por exemplo, a poluição dos recursos hídricos (COSTA, 2011).

Há grande relação e interferências entre todos os sistemas e serviços de saneamento básico no ambiente urbano, tais como entre os de esgotamento sanitário e de resíduos sólidos, nos quais a presença excessiva de resíduos pode gerar problemas nas instalações e unidades operacionais de esgotos (NEVES; TUCCI, 2008). Não apenas por eventuais danos que possam ocasionar em bombas, tubulações, equipamentos ou unidades de tratamento, como também gerando uma demanda operacional importante no que se refere às limpezas, manutenções e no adequado gerenciamento e destinação desses resíduos que podem ser considerados segundo a Política Nacional de Resíduos Sólidos, instituída pela Lei $\mathrm{n}^{\circ}$ 12.305/2010, como resíduos de serviços públicos de saneamento básico.

A eficiente gestão dos serviços públicos de saneamento básico interfere positivamente na condução da gestão de recursos hídricos, uma vez que ao estar atendendo às cidades sem eventuais paradas ou colapsos nos equipamentos e unidades operacionais do sistema de esgotamento sanitário, os usuários de recursos hídricos ligados aos serviços de saneamento básico podem contribuir para a mitigação de impactos danosos ao meio ambiente e aos corpos receptores.

A educação ambiental e sanitária da população também pode estar ligada ao aparecimento indevido de resíduos sólidos nos sistemas de esgotamento sanitário, uma vez que, muitos materiais são lançados às instalações hidrossanitárias ou tubulações sem que seja a destinação mais adequada, muitas vezes não propositalmente, mas por desconhecimento.

A ausência ou precariedade de serviços de limpeza urbana e de coleta de resíduos nas cidades e o envio de águas pluviais urbanas indevidamente às unidades dos sistemas de esgotos sanitários também contribuem para a observação de resíduos nas instalações de esgotos (TUCCI, 1997).

O presente trabalho analisou que tipos de resíduos são comumente encontrados em algumas unidades operacionais de esgotamento sanitário do sistema Insular do município de Florianópolis - SC, os quais ficam retidos nos gradeamentos.

Pelo que se pôde observar na busca de referencial bibliográfico, não há muitos estudos científicos em nível nacional que retratem esses materiais grosseiros, exceto aqueles que buscaram avaliar o potencial de reciclagem ou reutilização de lodo e areia removido das unidades de tratamento preliminares.

Entende-se, portanto, que o presente trabalho contribui para fomentar respostas acerca da gestão desses resíduos, bem como da gestão dos serviços de saneamento, da necessidade de busca por alternativas tecnológicas, operacionais e de educação ambiental e sanitária que potencialize a operação adequada dessas unidades do Sistema de Esgotamento Sanitário - SES frente à ocorrência de resíduos sólidos.

\section{MATERIAL E MÉTODOS}




\section{Área de estudo}

O município deste trabalho foi Florianópolis, capital do Estado de Santa Catarina, localizado na Região Sul do Brasil, entre os paralelos $27^{\circ} 10^{\prime}$ e $27^{\circ} 50^{\prime}$ de latitude Sul e entre os meridianos $48^{\circ} 25^{\prime}$ e $48^{\circ} 35^{\prime}$ de longitude Oeste, ocupando área de $438,5 \mathrm{~km}^{2}$ (Prefeitura Municipal de Florianópolis, 2021), com população total estimada de 508.826 habitantes (IBGE, 2020).

O trabalho foi desenvolvido junto a duas estações elevatórias de esgotos (EEE) do Sistema de Esgotamento Sanitário - SES Insular, operado pela concessionária estadual, a Companhia Catarinense de Águas e Saneamento - CASAN (Figura 1). O SES Insular abrange a porção central da llha de Santa Catarina, Distrito Administrativo Sede, sendo os bairros atendidos: Centro, Agronômica, Maciço do Morro da Cruz, José Mendes, Prainha, Saco dos Limões, Costeira do Pirajubaé, Pantanal, Córrego Grande, Itacorubi, Santa Mônica, Trindade (Prefeitura Municipal de Florianópolis, 2021).

Figura 1. Sub-bacias do SES Insular e unidades operacionais de estudo (EEE).

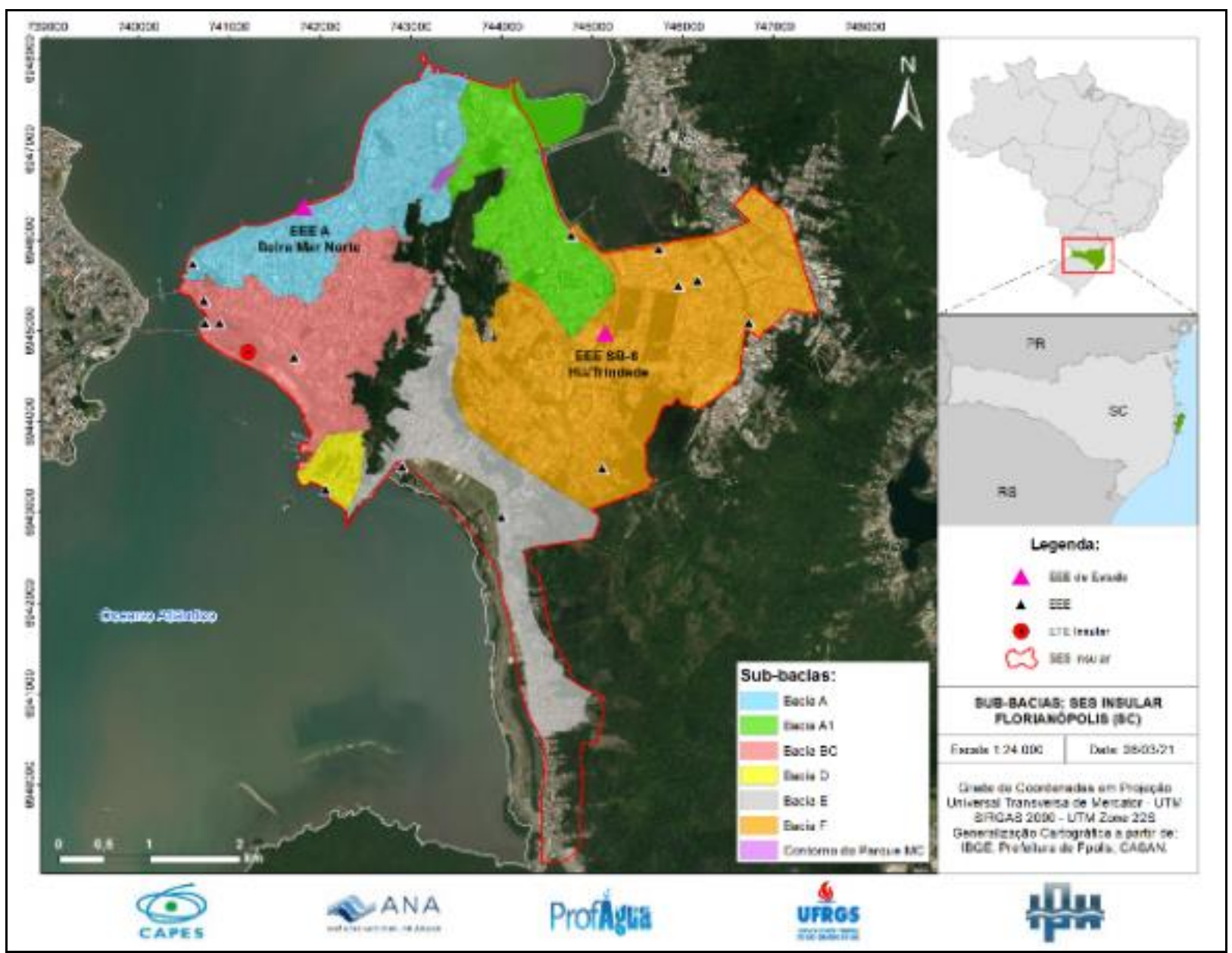

A população atendida pelo sistema é de cerca de 138.519 habitantes com 15.291 ligações e 59.296 economias. Possui 27 EEE e a vazão atual de tratamento é de 278,23 
Revista Brasileira de Ciência, Tecnologia e Inovação

L/s por meio da ETE Insular por processo de Lodos Ativados com aeração prolongada. O destino do efluente tratado é o mar junto à Baía Sul (Pref. Mun. de Florianópolis, 2021).

As EEE do SES Insular estudadas foram:

(i) EEE HU/Trindade (EEE SB6): inserida na Bacia de esgoto F, localizada aos fundos do Hospital Universitário Polydoro Ernani de São Thiago (HU) da Universidade Federal de

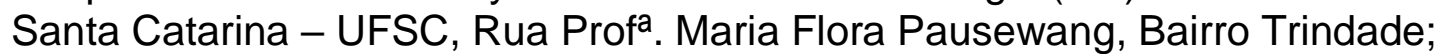

(ii) EEE Beira Mar Norte (EEE A): inserida na Bacia de esgoto A do sistema, localizada junto ao bolsão da Avenida Jornalista Rubens de Arruda Ramos, Bairro Centro, na Praça do Seisquicentenário da Polícia Militar.

Na Figura 2 tem-se visão local das EEE e dos gradeamentos instalados em cada unidade. A EEE HU/Trindade (EEE SB6) possui apenas uma unidade de gradeamento e a EEE Beira Mar Norte (EEE A), duas.

Figura 2. Vista Externa e dos Gradeamentos das Unidades Operacionais de estudo: (A) EEE HU/Trindade - SB6; (B) EEE Beira-mar - A.
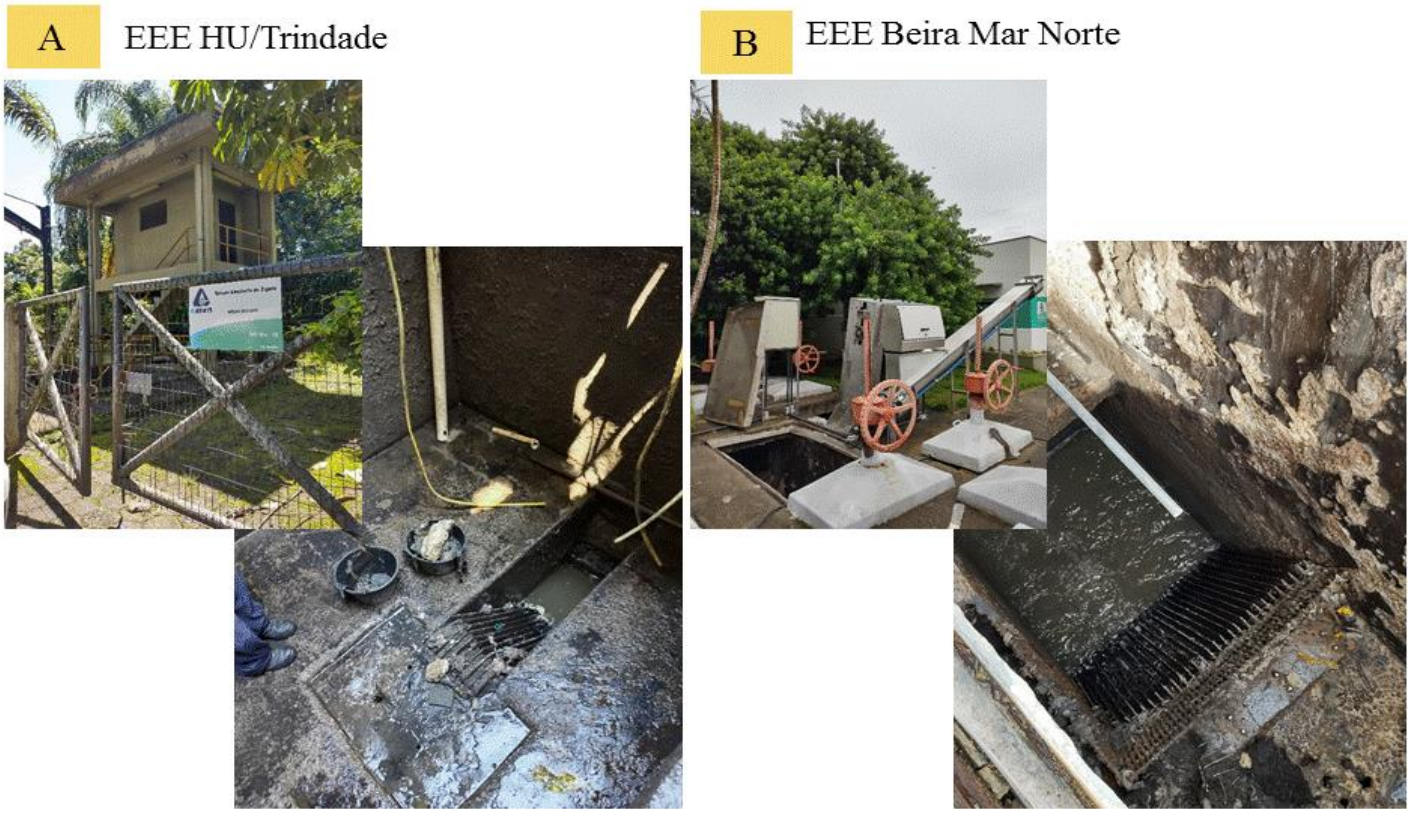

\section{Desenvolvimento do Estudo}

O presente estudo baseou-se no uso de método de análise de composição gravimétrica para identificar os principais resíduos sólidos grosseiros retidos nas estruturas de gradeamento das unidades operacionais do sistema Insular.

A metodologia básica seguiu as orientações técnicas do Instituto Brasileiro de Administração Municipal - (IBAM, 2001) para estudos de caracterização gravimétrica de resíduos sólidos urbanos, adaptando-as às condições dos resíduos que foram retirados das unidades de gradeamento das elevatórias do SES. Na Figura 3 apresenta-se uma composição com fotografias numeradas que mostram aspectos da metodologia desse trabalho.

Assim, foram realizadas as etapas de: coletar as amostras nos serviços de limpeza do gradeamento (01); transportar os resíduos em bombonas plásticas até o local cedido pela CASAN para desenvolvimento das atividades (02); espalhar o material sobre lona em 
Revista Brasileira de Ciência, Tecnologia e Inovação

área plana (03); separar os resíduos em cada uma das classes previamente escolhidas e classificar como "outros" os materiais encontrados que não se enquadrem na listagem pré-selecionada ou que não possam ser identificados visualmente (04); pesar cada componente separadamente (05); pesar a amostra total analisada (06). Posteriormente, os dados foram compilados, onde se dividiu o peso $(\mathrm{kg})$ de cada componente pelo peso total da amostra $(\mathrm{kg})$ e se pôde calcular a composição gravimétrica em termos percentuais.

Figura 3. Exemplicação das principais etapas do estudo gravimétrico (neste caso a imagem 01 é da retirada de resíduos do gradeamento na EEE HU/Trindade).

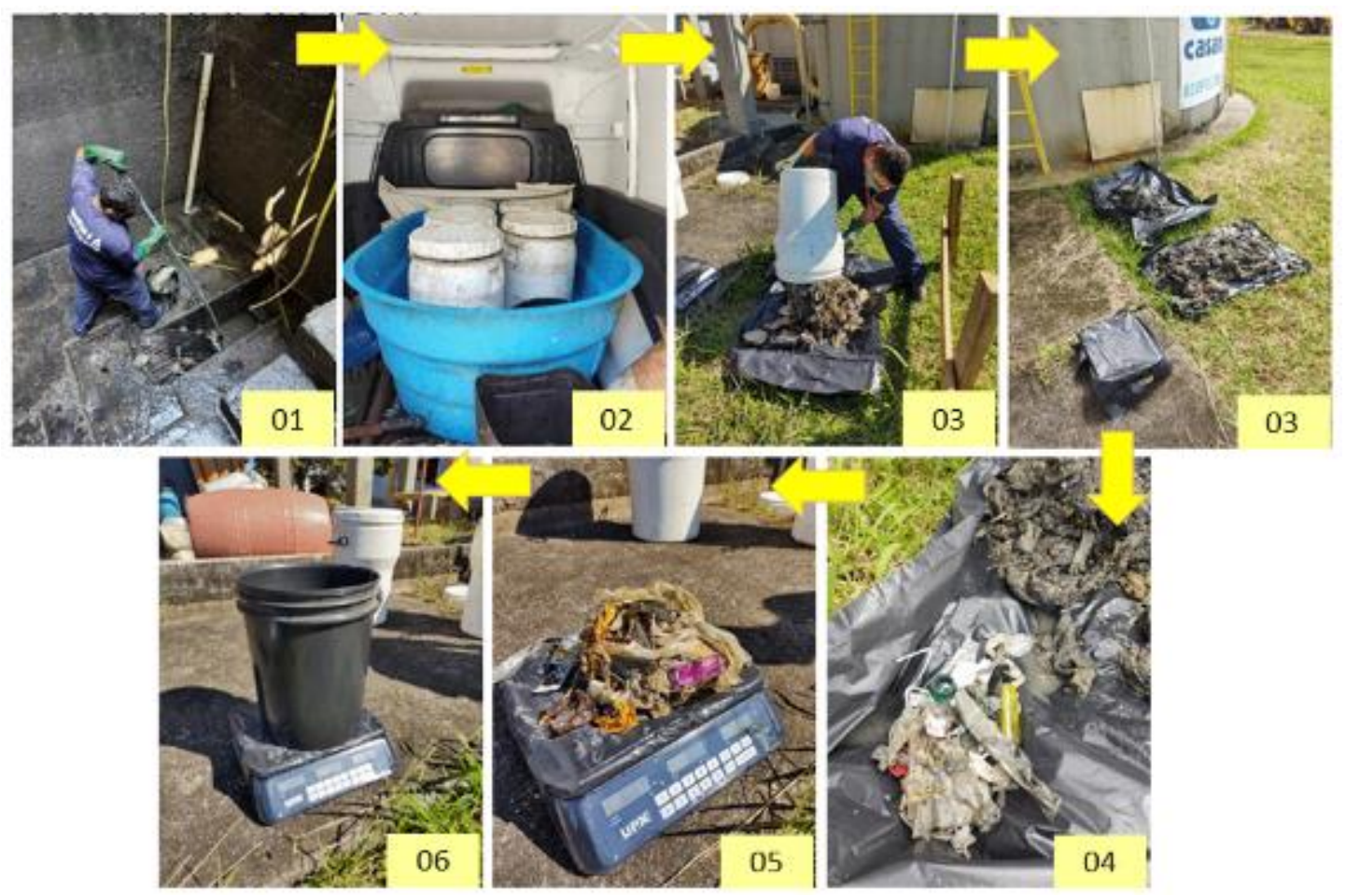

Definiram-se os principais componentes que se pretendeu determinar, ou seja, as classes dos materiais. Nesse caso, o trabalho apresentado por Morgado e Inácio (2014) foi adotado como referência, principalmente por ser um dos únicos trabalhos em nível nacional que verificaram os resíduos sólidos grosseiros encontrados em unidades do SES do Estado de São Paulo. Entretanto, não foi possível adotar todas as classes igualmente daquele, o qual inclusive contou com análises laboratoriais. Contudo, as principais classes definidas foram selecionadas para uso nesse trabalho e são mostradas conforme Tabela 1.

A análise gravimétrica foi realizada com a massa total úmida de resíduos grosseiros retirados dos gradeamentos nas atividades de limpeza operacional das unidades do sistema escolhido. Os resíduos grosseiros foram removidos dos gradeamentos pelo(s) funcionário(s) da própria operadora do SES, a CASAN, em sua rotina de manutenções operacionais normais. A quantidade total de material estudado refere-se ao total removido das unidades em um dia de manutenção, ou seja, o acumulado da limpeza realizada nas unidades que ocorre uma ou até duas vezes 
Revista Brasileira de Ciência, Tecnologia e Inovação

diariamente. Foram coletados um dia antes do estudo para fins de remoção natural (secagem) do excesso de esgoto líquido presente no material.

Tabela 1. Classes adotadas para os resíduos sólidos grosseiros

\begin{tabular}{ll}
\hline Classe & Resíduos \\
\hline Plásticos e látex & sacolas, embalagens de alimentos, preservativos etc. \\
\hline Plásticos moldados & embalagens, garrafas, tampinhas, recipientes, cotonetes etc. \\
Material de construção civil & pedaços cimento, madeiras, tijolos quebrados etc. \\
Animais mortos e similares & fetos, ratos etc. \\
Trapos e panos & roupas velhas, esponjas, tecidos em geral. \\
Matéria orgânica & gordura, folhas etc. \\
Outros e/ou rejeitos & materiais que não possam ser identificados ou segregados ou \\
& que já estejam em avançado estado de putrefação etc. \\
\hline
\end{tabular}

Fonte: Baseado em Morgado e Inácio (2014).

A quantidade integral dos resíduos grosseiros foi pesada e manipulada na quantia retirada das unidades operacionais, ou seja, não houve quarteamento das amostras. Todos os resíduos grosseiros foram identificados e pesados, sendo que a quantidade total de cada dia de levantamento também foi pesada.

As amostragens foram realizadas em frequência quinzenal nas unidades, após a remoção dos materiais dos gradeamentos. É relevante salientar que esse trabalho se encontra em desenvolvimento e que as amostragens continuarão a ser coletadas até Julho/2021, entretanto os primeiros resultados são apresentados nesta publicação compreendendo o período de amostragem de Fevereiro até Abril/2021.

\section{RESULTADOS E DISCUSSÕES}

Os resultados preliminares desse trabalho são mostrados na Tabela. 2 considerando-se as classes adotadas. Observando-se as amostragens individualmente há bastante variação seja no peso total dessa, seja nas características dos resíduos sólidos categorizados nas classes predefinidas. Assim, um maior período amostral é justificado nesse trabalho para que se possa analisar variabilidades ao longo do tempo.

Nesse trabalho os "rejeitos e outros" foram considerados todos aqueles que não podem ser visualmente caracterizados e por consequência manualmente separados. $O$ que se observou em termos qualitativos é que o material retido nas unidades de gradeamento já se apresentou em um avançado estado de putrefação e que houve a ocorrência excessiva de fibras têxteis que compõe principalmente os lenços umedecidos utilizados pela população. Estes materiais formam uma massa compacta de difícil manuseio aderido a outras substâncias orgânicas.

De acordo com Morgado e Inácio (2014) a ocorrência dessas fibras foi de cerca de $26,4 \%$ da composição dos materiais retidos nas grades médias. No presente trabalho, observou-se visualmente que esses materiais foram a maior parte dos materiais observados na classe de "rejeitos e outros", porém sem que houvesse possibilidade real de segregá-los minuciosamente. Os lenços umedecidos são comumente formados por TNT, ou seja, "tecido não tecido", composto por alguma fibra de origem vegetal, que pode ser a viscose ou poliéster e plásticos. 
RBCTI

Tabela 2. Resultados iniciais da gravimetria das EEE de estudo (Fev. a Abril/2021)

\begin{tabular}{|c|cccccccccc|c|c|}
\hline \multicolumn{10}{c|}{ EEE HU/Trindade - SB6 } & \multicolumn{1}{c|}{ Totais } \\
\hline Peso (kg) por Classe & $04 / 02$ & $\%$ & $18 / 02$ & $\%$ & $04 / 03$ & $\%$ & $18 / 03$ & $\%$ & $01 / 04$ & $\%$ & $\mathrm{~kg}$ & $\%$ \\
\hline Plásticos/Látex & 0,65 & 2,63 & 0,42 & 3,64 & 0,73 & 5,42 & 0,02 & 0,20 & 0,08 & 0,89 & 1,91 & 2,7 \\
\hline Plásticos Moldados & 0,08 & 0,31 & 0,06 & 0,55 & 0,10 & 0,75 & 0,10 & 0,89 & 0,04 & 0,43 & 0,38 & 0,5 \\
\hline RCC & 0,08 & 0,33 & - & - & - & - & - & - & - & - & 0,08 & 0,1 \\
\hline Animais Mortos & - & - & - & - & - & - & - & - & - & - & - & - \\
\hline Trapos e Panos & - & - & - & - & - & - & & - & - & - & - & - \\
\hline Matéria Orgânica & 4,57 & 18,40 & 0,50 & 4,29 & - & - & 0,25 & 2,22 & - & - & 5,32 & 7,5 \\
\hline Rejeitos e outros & 19,45 & 78,32 & 10,67 & 91,53 & 12,57 & 93,84 & 10,88 & 96,69 & 9,28 & 98,68 & 62,85 & 89,1 \\
\hline Total Amostra $\mathbf{( k g )}$ & $\mathbf{2 4 , 8 3}$ & $\mathbf{1 0 0 , 0 0}$ & $\mathbf{1 1 , 6 6}$ & $\mathbf{1 0 0 , 0 0}$ & $\mathbf{1 3 , 4 0}$ & $\mathbf{1 0 0 , 0 0}$ & $\mathbf{1 1 , 2 5}$ & $\mathbf{1 0 0 , 0 0}$ & $\mathbf{9 , 4 0}$ & $\mathbf{1 0 0 , 0 0}$ & $\mathbf{7 0 , 5 4}$ & $\mathbf{1 0 0 , 0}$ \\
\hline
\end{tabular}

\begin{tabular}{|c|cccccccccc|c|c|}
\hline \multicolumn{11}{c|}{ EEE Beira Mar Norte - A } \\
\hline Peso (kg) por Classe & $04 / 02$ & $\%$ & $18 / 02$ & $\%$ & $04 / 03$ & $\%$ & $18 / 03$ & $\%$ & $01 / 04$ & $\%$ & $\mathrm{~kg}$ & $\%$ \\
\hline Plásticos/Látex & 0,03 & 0,12 & 0,31 & 2,34 & 0,43 & 2,62 & 0,08 & 0,69 & 0,40 & 1,66 & 1,25 & 1,4 \\
\hline Plásticos Moldados & - & - & - & - & - & - & 0,03 & 0,22 & 0,15 & 0,61 & 0,17 & 0,2 \\
\hline RCC & - & - & - & - & - & - & - & - & - & - & - & - \\
\hline Animais Mortos & - & - & - & - & - & - & - & - & 0,12 & 0,50 & 0,12 & 0,1 \\
\hline Trapos e Panos & 0,06 & 0,24 & 0,15 & 1,13 & 0,04 & 0,24 & - & - & - & - & 0,25 & 0,3 \\
\hline Matéria Orgânica & - & - & 0,25 & 1,86 & - & - & 2,21 & 19,05 & 0,57 & 2,37 & 3,03 & 3,3 \\
\hline Rejeitos e outros & 25,22 & 99,64 & 12,54 & 94,67 & 15,93 & 97,13 & 9,28 & 80,03 & 22,86 & 94,85 & 85,83 & 94,7 \\
\hline Total Amostra (kg) & $\mathbf{2 5 , 3 1}$ & $\mathbf{1 0 0 , 0 0}$ & $\mathbf{1 3 , 2 5}$ & $\mathbf{1 0 0 , 0 0}$ & $\mathbf{1 6 , 4 0}$ & $\mathbf{1 0 0 , 0 0}$ & $\mathbf{1 1 , 6 0}$ & $\mathbf{1 0 0 , 0 0}$ & $\mathbf{2 4 , 1 0}$ & $\mathbf{1 0 0 , 0 0}$ & $\mathbf{9 0 , 6 6}$ & $\mathbf{1 0 0 , 0}$ \\
\hline
\end{tabular}

É importante ressaltar a ocorrência desses materiais nas redes de esgotos e por consequência nas unidades de gradeamento, pois não são de fácil decomposição como o papel higiênico e sim, trata-se de materiais ou resíduos que deveriam ser encaminhados às lixeiras e destinados ao sistema de manejo de coleta de resíduos sólidos do município e não ao SES.

O mesmo diz respeito aos plásticos e látex observados nas amostragens, demonstrando a pouca conscientização da população quanto aos materiais que são enviados às instalações hidrossanitárias ligadas às redes de esgotos.

Convém, contudo salientar que há possibilidade de parte desses plásticos e outros materiais como os RCC, os quais chegam às redes coletoras de esgotos devido à interferências do sistema de drenagem urbana pluvial e ligações indevidas, bem como ausência ou precariedade na disponibilização de serviços públicos de limpeza urbana ou de coleta de resíduos sólidos urbanos em espaços urbanos. Isto contribui para o acúmulo de resíduos em calçadas, vias e outros ambientes que podem alcançar as unidades do sistema de esgotamento sanitário devido ao carreamento dos mesmos pela chuva (POMPÊO, 2000; TUCCI, 2002; CASTILHO JÚNIOR, 2003; BERTOLINO et al., 2018).

Neves e Tucci (2008) indicaram que os serviços de limpeza urbana são aqueles de maior relação com a presença de resíduos na drenagem urbana, tendo em vista que são mais suscetíveis a atingí-la. Por consequência, tem-se que a presença de resíduos sólidos nas unidades de esgotamento sanitário pode estar relacionada ao envio de águas pluviais ao sistema de esgoto.

Tucci (1997) menciona que no Brasil a maioria das redes de coleta de esgotos é conceitualmente empregada como separador absoluto, ou seja, havendo distinção entre o manejo das águas pluviais e dos esgotos sanitários, ou seja, sistemas operacionais independentes. Já Pompêo (2000) discorre que "raramente" há distinção entre o uso de 
Revista Brasileira de Ciência, Tecnologia e Inovação

sistemas de drenagem pluvial e de esgotamento sanitário. Isto porque, mesmo Tucci (1997) e Pompêo (2000) concordam, é a falta de investimentos em estruturas de esgotamento sanitário nas cidades que acaba por fazer com que os sistemas passem a ser de uso misto, mesmo que conceitualmente não tenham sido de fato concebidos com essa finalidade.

Nos resultados, até o presente momento, observou-se pouca ocorrência das classes de resíduos da construção civil (RCC), animais mortos, trapos e panos, tendo sido esses materiais observados apenas em algumas amostragens.

Os plásticos moles e látex, que puderam ser segregados manualmente, foram observados com relevante ocorrência nas duas unidades operacionais, sendo cerca de $2,7 \%$ do total na EEE HU/Trindade e 1,4\% na EEE Beira Mar Norte. A matéria orgânica que nesse trabalho correspondeu principalmente à segregação e pesagem dos flocos de gordura desprendidos das tubulações do sistema que ficam retidas nos gradeamentos, resultou na classe mais relevante, cerca de $7,5 \%$ no total observado na EEE HU/Trindade e, 3,3\% na EEE Beira Mar Norte.

Consultando-se o trabalho de Morgado e Inácio (2014), para as grades médias estudadas, os tecidos (trapos e panos), as gorduras e os plásticos moles e moldados também foram os de maior ocorrência em termos percentuais, sendo, respectivamente: $30,1 \%, 9,5 \%, 16,4 \%$ e $14,9 \%$. Já nesse trabalho os plásticos moldados, por exemplo, foram pouco ocorrentes até o presente momento, o que tende a ser positivo, uma vez que esses materiais realmente não deveriam ser observados nas redes de esgotos.

Assim, entende-se que com o desenvolvimento desse estudo será possível verificar a ocorrência das tipologias dos resíduos observados no SES Insular, podendo-se determinar a possível origem desses e, se há diferenças entre as duas unidades em razão dos resultados a serem obtidos para cada uma delas, apesar de fazerem parte deste SES.

Esses dados poderão auxiliar na tomada de decisão para o gerenciamento de resíduos sólidos no sistema, fomentando a revisão do(s) planos de gerenciamento de resíduos sólido - PGRS das unidades operacionais, bem como promovendo-se um olhar sobre as parcelas de resíduos encontrados que vise atender às diretrizes de gestão da Política Nacional de Resíduos Sólidos - PNRS (Lei N 12.305/2010).

O que já se observou é que a ocorrência de resíduos sólidos nas unidades do SES Insular estudadas, os quais não deveriam ser ocorrentes em excesso, tais como os plásticos moles, látex, plásticos moldados e os resíduos caracterizados como lenços umedecidos demonstram deficiência de ações concretas para a educação ambiental e sanitária da população em planos setoriais, como os de saneamento básico, gestão de resíduos sólidos e de recursos hídricos.

\section{CONCLUSÕES}

Os resultados dos resíduos sólidos observados nas unidades operacionais SES Insular desencadeiam olhar sobre as políticas de saneamento básico e de gestão de recursos hídricos, uma vez que, problemas nessas unidades ocasionadas por resíduos sólidos, os quais não deveriam ser encontrados em excesso, resultam em ocorrências que podem impactar negativamente a qualidade ambiental e sanitária do ambiente urbano e dos corpos receptores, da drenagem urbana pluvial etc.

A deficiência de instrumentos e de ações concretas para educação ambiental e sanitária, bem como para gestão dos serviços de saneamento, tais como nos planos 
Revista Brasileira de Ciência, Tecnologia e Inovação

setoriais (saneamento, recursos hídricos, resíduos sólidos), indicam a necessidade de propor alternativas específicas que possam integrar essas temáticas dirimindo potenciais danos e impactos negativos associados a elas.

Por isso, o presente trabalho por meio desses resultados iniciais mostrou que há necessidade de se definir meios de conscientizar a população sobre o adequado gerenciamento dos resíduos sólidos gerados nas edificações e sua relação com as instalações hidrossanitárias ligadas ao SES.

\section{AGRADECIMENTOS}

O presente trabalho foi realizado com apoio da Coordenação de Aperfeiçoamento de Pessoal Nível Superior - Brasil (CAPES) - Código de Financiamento 001. Agradeço também ao Programa de Mestrado Profissional em Rede Nacional em Gestão e Regulação de Recuros Hídricos - ProfÁgua, Projeto CAPES/ANA AUXPE n²717/2015 e à Companhia Catarinense de Águas e Saneamento (CASAN) pelo apoio técnico e científico aportado até o momento.

\section{REFERÊNCIAS}

BERTOLINO, M., KONDAGESKI, J. H., WEINSCHUTZ, R. Água de Chuva domiciliar no esgoto separador absoluto. Revista DAE, v. 66, n. 213, p. 100-108. 2018.

CASTILHO JUNIOR, A. B. (org). Resíduos Sólidos Urbanos: Aterro Sustentável para Municípios de Pequeno Porte. Rio de Janeiro: ABES, 2003.

COSTA, S. L. Gestão Integrada de resíduos sólidos: aspectos jurídicos e ambientais. Aracaju: Evocati. 2011.

INSTITUTO BRASILEIRO DE ADMINISTRAÇÃO MUNICIPAL (IBAM). Manual de Gerenciamento Integrado de Resíduos Sólidos. José Henrique Penido Monteiro (Coordenador). Rio de Janeiro. Instituto Brasileiro de Administração Municipal (IBAM). 2001. $204 \mathrm{p}$.

INSTITUTO BRASILEIRO DE GEOGRAFIA E ESTATÍSTICA (IBGE). Estimativas da população residente para os municípios e para as unidades da federação brasileiros com data de referência de 1 de Julho de 2020. Rio de Janeiro. 2020. Disponível em: https://cidades.ibge.gov.br/brasil/sc/florianopolis/panorama. Acesso em: 22 mar. 2021.

MORGADO, M.; INACIO, G. Caracterização da composição de resíduos removidos em gradeamento de ETEs. Revista Hydro, v. XII, n. 89. p.16-27. 2014.

NEVES, M. G. F. P.; TUCCI, C. E. M. Resíduos Sólidos na Drenagem Urbana: Aspectos Conceituais. Revista Brasileira de Recursos Hídricos, v. 13, n. 3, p. 125-135, 2008.

POMPÊO, C. A. Drenagem Urbana Sustentável. Revista Brasileira de Recursos Hídricos, v. 5, n. 1, p. 15-24, 2000. 
Revista Brasileira de Ciência, Tecnologia e Inovação

PREFEITURA MUNICIPAL DE FLORIANÓPOLIS. Revisão do Plano Municipal Integrado de Saneamento Básico (PMISB) de Florianópolis. - Versão Preliminar. Secretaria Municipal de Infraestrutura. Jan. 2021. 251 p. Disponível em:

http://portal.pmf.sc.gov.br/entidades/infraestrutura/index.php?cms=consulta+publica+pmis b+2021\&menu=0. Acesso em: 11 fev. 2021.

TUCCI. C. E. M. Água no Meio Urbano. In: Água Doce. Cap. 14. Porto Alegre: IPH/UFRGS. 1997.

TUCCI, C. E. M. Gerenciamento da Drenagem Urbana. Revista Brasileira de Recursos Hídricos, v. 7, n. 2, p. 5-27, 2002. 